\title{
CONCEPTION ET SIMULATION D’UN MICRO-CAPTEUR A ONDES DE LOVE PAR ELEMENTS FINIS
}

\author{
Hamida HalliL ${ }^{1 *}$, Prince Bahoumina ${ }^{1}$, Ollivier TAMARIN ${ }^{2}$, Corinne DeJous ${ }^{1}$, Dominique RebIERE ${ }^{1}$ \\ Université de Bordeaux, Bordeaux INP, Laboratoire IMS, CNRS UMR 5218 - 351 Cours de la \\ Libération ; 33405 Talence Cedex, France \\ Univesité des Antilles et de la Guyane, UMR ESPACE DEV, IRD - 0.275 Km Route de Montabo ; BP 165 - \\ 97323 Cayenne cedex, France
}

E-mails : *hamida.hallil-abbas@u-bordeaux.fr, prince.bahoumina@u-bordeaux.fr, ollivier.tamarin@guyane.univag.fr, corinne.dejous@ims-bordeaux.fr, dominique.rebiere@u-bordeaux1.fr.

Résumé : Cette communication présente un tutoriel proposé à des étudiants stagiaires de licences professionnelles ou masters relevant des nanosciences. Il s'agit de modéliser et de simuler un capteur à onde de Love en $3 D$, pour la détection in-situ de particules chimiques ou biologiques dans un milieu gazeux ou liquide. Les étudiants sont ainsi initiés à la fois à la modélisation d'un capteur dans un environnement à éléments finis, à la modélisation de son environnement de travail et ainsi qu'à son application pour la détection d'espèces gazeuses et son utilisation en milieu liquide pour des applications environnementales.

Mots clés : Modélisation d'un capteur de gaz, micro-capteur à onde acoustique, SAW, COVs, PDMS.

1. Présentation : La Pollution de l'air et de l'eau est responsable du décès de millions de personnes dans le monde. Pourtant, peu de capteurs chimiques sont présents aujourd'hui sur le marché. Le défi pour stimuler le renouveau industriel lié à cette problématique de mesure et instrumentation est la recherche de capteurs fiables, à faible consommation, à bas cout, à forte sensibilité et sélectivité. Dans le cadre de cette formation, nous proposons aux étudiants la modélisation d'une plateforme acoustique associée à un matériau sensible, favorisant un effet de masse particulièrement important dû à leur grande surface d'interaction avec des espèces cibles. Notre approche de simuler ce capteur par éléments finis offre une facilité d'optimisation de la géométrie de la plate-forme et de la prise en compte des propriétés des matériaux utilisés pour la conception d'un capteur ultrasensible et sélectif dédié à la détection rapide de composés chimiques à l'état de vapeur ou en milieu liquide. En effet, l'utilisation des outils de modélisation multiphysiques à éléments finis permettent aux étudiants d'avoir les bases suffisantes pour appréhender les résultats expérimentaux obtenus après fabrication et caractérisation des capteurs réels et les confronter à des modèles théoriques.

Le dispositif étudié est un capteur à onde de Love configuré en ligne à retard résonante. La ligne à retard présentée en figure 1 possède deux IDTs, placées de part et d'autre de la couche sensible. L'IDT émetteur sert à générer l'onde acoustique et l'IDT récepteur permet de reconvertir l'onde acoustique en un signal électrique. Le temps que met l'onde pour traverser la distance (Gap) qui sépare les deux IDTs donne son nom au dispositif [1-2]. C'est un modèle simplifié du capteur réel qui était simulé pour obtenir les différents paramètres caractéristiques tels que la polarisation de l'onde, l'amplitude des déplacements, la profondeur de pénétration de l'onde dans le substrat et l'influence de l'effet de masse sur l'onde. L'une des particularités de ce modèle est sa réversibilité, du fait de sa symétrie. Aussi le signal reçu sur l'IDT récepteur est retardé et atténué par rapport au signal émis, d'où l'appellation de ligne à retard [2]. Son coefficient de qualité dépend du nombre de doigts constituant chaque IDT et 
la distance entre chaque doigt est définie pour avoir un phénomène cumulatif d'ondes constructives à la fréquence d'excitation.

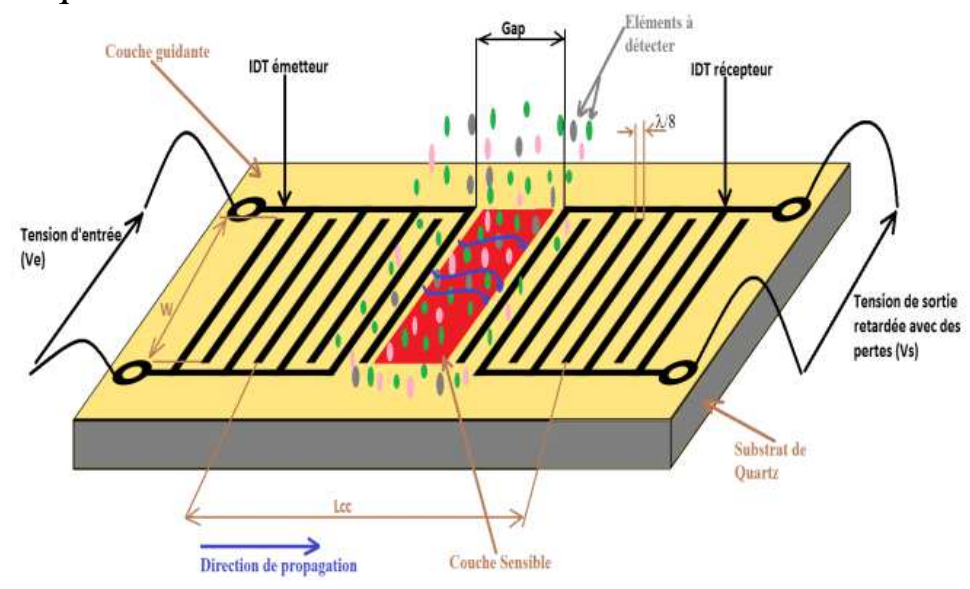

Figure 1. Principe de fonctionnement du capteur

Le principe de détection du capteur est basé sur la variation des paramètres internes ou externes des matériaux qui le constituent (masse volumique, élasticité, viscosité, épaisseur des couches, température, pression), susceptibles de perturber les caractéristiques de l'onde lors de sa propagation [2]. Par exemple, sa vitesse de propagation ou son amplitude. Dans notre cas c'est la masse volumique qui influe sur l'onde, puisque les études sur les dispositifs à ondes acoustiques montrent que les caractéristiques de propagation de l'onde sont fortement liées à la masse volumique des matériaux intervenant dans la structure considérée (le substrat, la couche guidante et la couche sensible). La présence des particules cibles sur la couche sensible qui se situe sur le chemin acoustique engendre un effet de masse et par conséquence, perturbe l'onde (figure 1). La mesure précise de ces perturbations, nous permet de détecter des particules spécifiques et de remonter à leur concentration liée au pourcentage de l'effet de masse. L'utilisation du capteur pour la détection de gaz se fait dans un boitier hermétique et la génération contrôlée d'espèces gazeuses se fait avec un générateur de vapeur. En revanche, l'utilisation du dispositif dans un milieu liquide nécessite une protection des IDTs. Pour cela, on utilise une puce PDMS (polydiméthylsiloxane) micro-fluidique.

2. Dispositif sans PDMS : Le capteur réel (figure2) est constitué de deux lignes à retard parallèles. Il a sur les IDTs de chaque ligne 88 doigts espacés de $5 \mu \mathrm{m}$ soit $\lambda / 8$. L'une des lignes a une couche sensible ayant des affinités avec les particules à détecter.

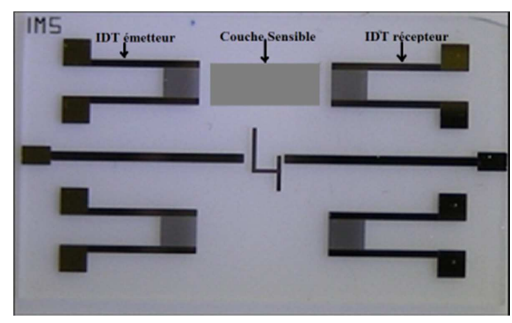

Figure 2. Capteur SAW réel sans PDMS

2.1. Présentation du dispositif simulé : Le modèle simulé (figure3) est composé d'un substrat de quartz en coupe AT (angles d'Euler : $0^{\circ} ; 121,5^{\circ} ; 90^{\circ}$ ) sur lequel est déposé les 
IDTs en Or, suivi d'une couche guidante isotrope en $\mathrm{SiO}_{2}$ et d'une couche sensible en $\mathrm{TiO}_{2}$ méso-poreux. Cette structure représente uniquement l'une des lignes avec 88 doigts sur chaque IDTs, espacés de $5 \mu \mathrm{m}$ également.

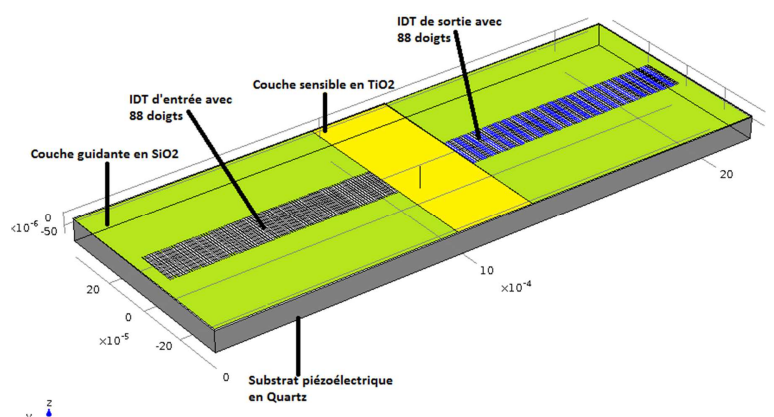

Figure 3. Capteur simulé sans PDMS

En vue de réduire les réflexions, la structure est à doubles doigts c'est-à-dire les doigts sont inter-digités deux par deux (comme on peut le voir dans la figure 3 avec les doigts actifs de l'IDT de sortie en bleu) mais cela ne suffit pas totalement comme le montreront les résultats. Sur l'IDT de sortie en trouve en bleu les doigts non reliés à la masse sur lesquels on récupère le potentiel électrique. La modélisation de la présence d'éléments à détecter se fait par un effet de masse de variation de 5\% sur la couche sensible [3-4]. Le signal électrique (impulsionnel ou sinusoïdal) est appliqué sur l'IDT d'émission situé à la gauche de la figure 3, pour générer l'onde de Love grâce à l'effet piézo-électrique. L'onde se déplace dans la direction $\mathrm{X}$ avec un maximum d'énergie confinée dans la couche guidante. Après un certain temps (temps de retard), l'onde acoustique arrive sur l'IDT de sortie et elle est convertie en un signal électrique. Afin d'obtenir la réponse impulsionnelle du dispositif, nous avons choisi d'exciter l'entrée avec une impulsion définie par les potentiels V+ et V- appliqués alternativement sur les doigts doubles de l'IDT émetteur à la fréquence de 117 MHz (figure4).

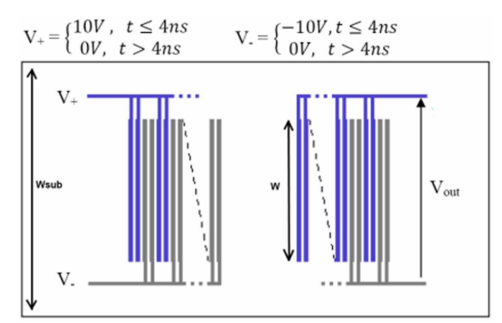

Figure 4. Affectation des potentiels d'excitation

2.2. Résultats de simulation sans PDMS : La structure est dimensionnée pour avoir le meilleur signal possible en sortie. Le déplacement de l'onde sur le dispositif avec son intensité sont présentés en figure 5. Pour voir la vidéo de la propagation de l'onde cliquez ici

Video_propagation_onde_sans-PDMS.gif

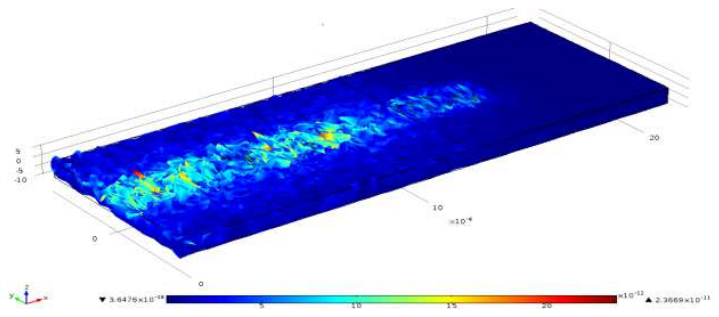

Figure 5. Propagation et intensité de l'onde sur la couche guidante à $0.1 \mu \mathrm{s}$ 
La propagation de l'onde est clairement visible dans le dispositif et la forme impulsionnelle du signal électrique appliqué est identifiable grâce aux pics de déformation mécanique de la structure. Nous retrouvons la composante Transverse Horizontale (TH) en vert, Transverse Verticale (TV) en rouge et Longitudinale (L) en bleu; en fonction du temps, lors du déplacement de l'onde au niveau de l'IDT récepteur (figure6). On peut voir que l'onde générée est quasi Transverse Horizontale pure puisque l'amplitude des deux autres composantes est presque nulle. Ce résultat confirme que l'onde générée est une onde de Love. Le temps d'attente avant l'apparition de l'onde en sortie correspondant au temps que met l'onde pour se déplacer de l'IDT d'entrée vers l'IDT de sortie du dispositif, soit un retard de 40 ns environ.

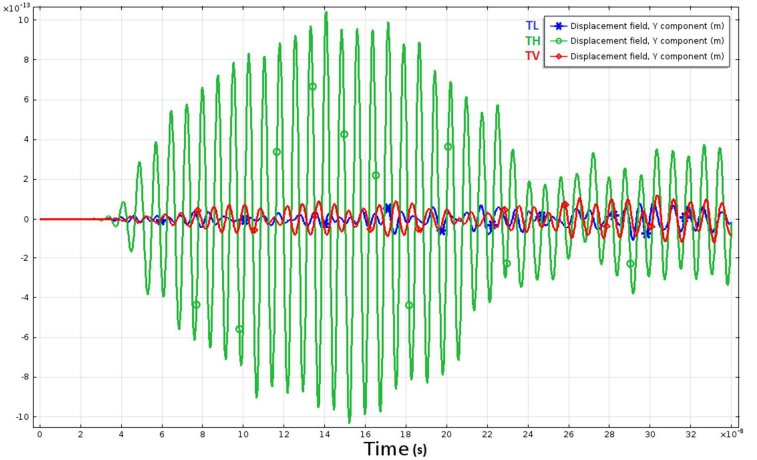

Figure 6. Déplacement de chaque composante de l'onde

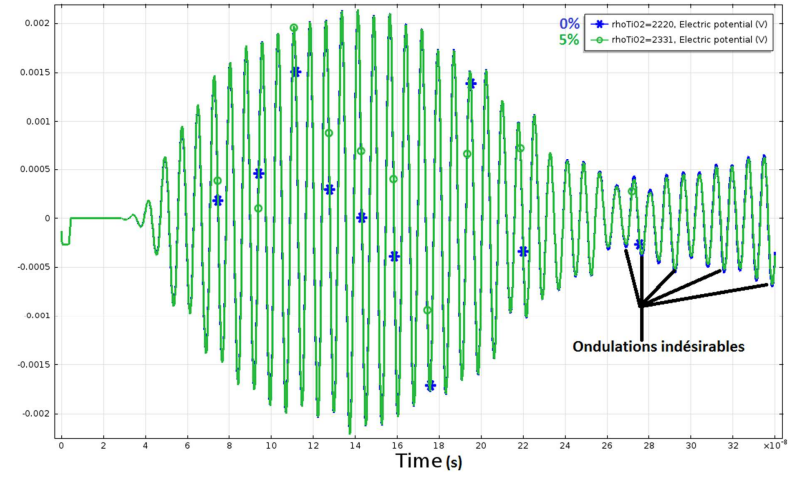

Figure 7. Réponse impulsionnelle de la structure pour $0 \%$ et $5 \%$ d'effet de masse

L'onde est principalement guidée en surface et les réponses impulsionnelles de la structure sont présentées en figure7. Elles montrent que nous récupérons à peine $2 \mathrm{mV}$ sur l'IDT récepteur. Ainsi le rendement en tension ne dépasse pas $0,02 \%$ et il y'a des ondulations indésirables, probablement causées par les réflexions de l'onde sur les parois du dispositif.

Pour remonter aux caractéristiques fréquentielles, nous exploitons les données des potentiels électriques en sortie du modèle, grâce à la transformée de Fourier. Cette structure à 21,7 dB de pertes d'insertion sans effet de masse et sa fréquence de résonnance est de $130 \mathrm{MHz}$ (figure8). L'onde met 14,1 $\mu$ s pour se déplacer entre le centre de chaque IDT, soit une vitesse de propagation de $8440 \mathrm{~m} / \mathrm{s}$. Le coefficient de qualité est de 27,1 pour une bande passante $\left(\mathrm{f}_{2}\right.$ $\mathrm{f}_{1}$ ) à $-3 \mathrm{~dB}$ de 4,8 $\mathrm{MHz}$. Nous avons (figure 9) des plages de variation linéaire de la phase dans la bande passante de 128,2 MHz à $133 \mathrm{MHz}$.

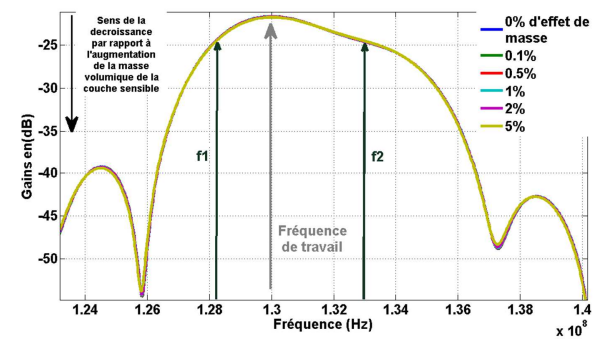

Figure 8. Gain de la structure

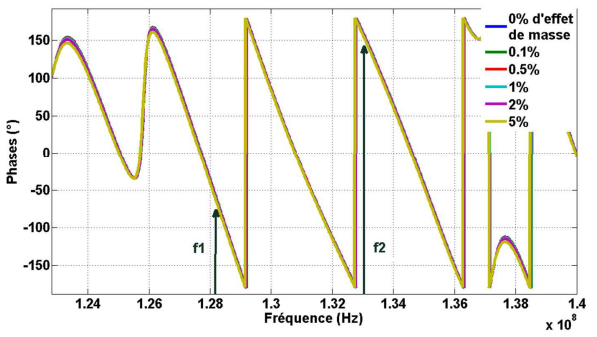

Figure 9. Phase de la structure

Nous avons défini le comportement du dispositif en présence d'un effet de masse de 0,1\% $0,5 \% 1 \% 2 \%$ et $5 \%$ pour établir la sensibilité. Lorsqu'on fait un zoom sur les courbes de phases autour de $0^{\circ}$, on remarque un décalage de la phase en fréquence (figure 10). 
La différence de fréquence liée à ce décalage est d'environ $0,5 \mathrm{KHz}, 2 \mathrm{KHz}, 5 \mathrm{KHz}, 10 \mathrm{KHz}$, $20 \mathrm{KHz}$ pour les différents effets de masse respectivement.

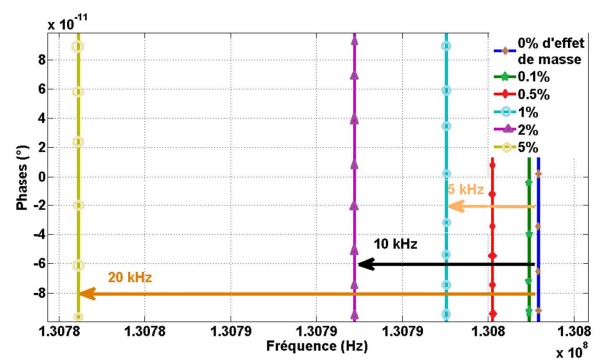

Figure 10. Variation de fréquence en fonction de l'effet de masse

3. Dispositif avec PDMS : Les réflexions qui engendrent des ondulations indésirables dans le dispositif précédent (figure 7) malgré, les IDTs à double doigts ont fait l'objet d'études et d'expériences diverses [5], et l'utilisation du PDMS donne des résultats concluants sur l'atténuation des réflexions [6-7]. De plus, le PDMS à une bonne adhésion sur du $\mathrm{SiO}_{2}$ [8], d'où son utilisation comme capot pour améliorer la réponse du dispositif et pour protéger les IDTs dans les applications qui nécessitent l'utilisation de la micro-fluidique (figure 11). Pour voir la vidéo d'une des applications de détection (de biomarqueurs bien spécifiques de présence des tumeurs cancéreuses) dans un milieu liquide à l'aide d'une puce micro-fluidique,

cliquez ici.

SAWaveccellulePDMS-detection de biomarqueurs.WMV

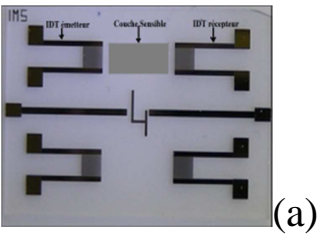

Figure 11. a) Capteur

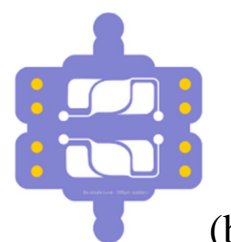

(b)

b) Empreinte de PDMS

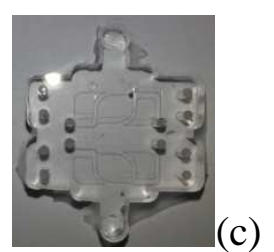

c) Capot de PDMS

3.1. Présentation du dispositif simulé : Le modèle simulé est identique à celui simulé sans PDMS, seule la couche de PDMS est rajoutée sur la couche guidante comme illustré en figure 12. Les paramètres de simulation reste inchangés également.

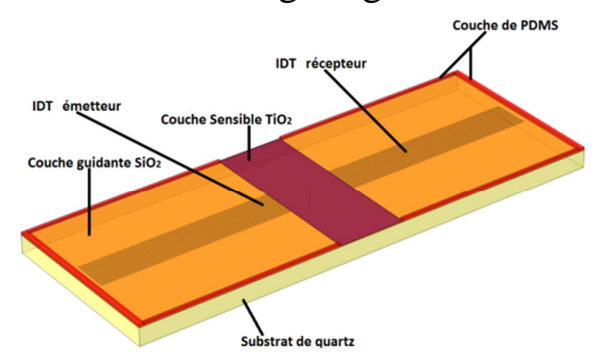

Figure 12. Capteur simulé avec PDMS

3.2. Résultats de simulation avec PDMS : Le PDMS est dimensionné puis positionné pour avoir le meilleur signal et meilleur rendement possible en sortie. Le déplacement de l'onde sur le dispositif avec son intensité sont visualisés à l'instant 0,45 ns (figure13). Pour voir la vidéo de la propagation de l'onde cliquez ici video-propagation_onde_evec_poms.gif 


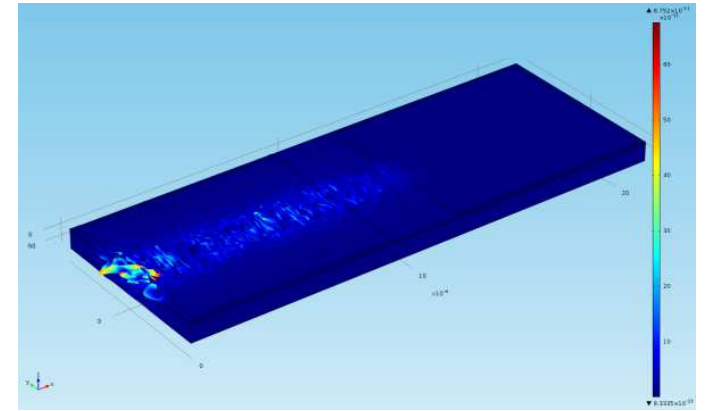

Figure 13. Propagation et intensité de l'onde sur la couche guidante à $0,45 \mathrm{~ns}$

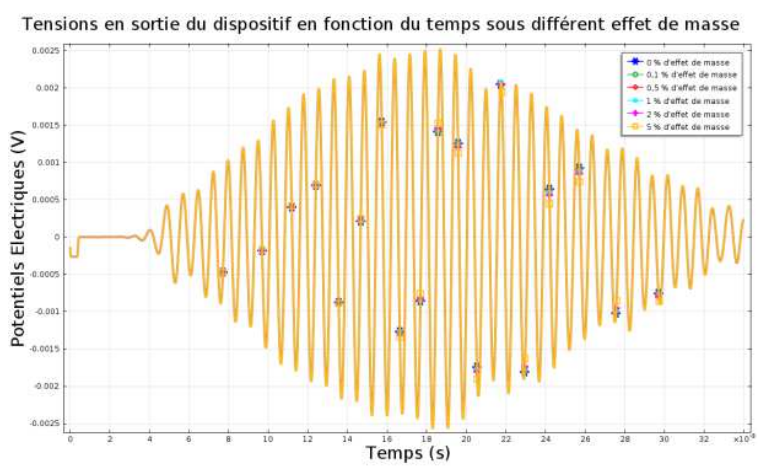

Figure 14. Réponse impulsionnelle sous effet de masse

L'onde générée est toujours Transverse Horizontale pure et son retard pour atteindre la sortie reste de $40 \mathrm{~ns}$, mais sa polarisation dépend désormais aussi de la surface et de l'épaisseur du PDMS. En outre l'onde reste guidée en surface. Les réponses impulsionnelles sont améliorées en termes d'allures et d'amplitudes avec l'élimination des ondulations indésirables (figure 14). Mais le rendement en tension reste faible, à peine $0.025 \%$. L'amélioration se traduit aussi par la réduction des pertes à 19,06 dB sans effet de masse et un décalage de la fréquence de résonnance à $130.6 \mathrm{MHz}$ (figure15). Le temps de propagation de l'onde augmente de14,1 $\mu$ s à $18.68 \mu \mathrm{s}$, ce qui réduit la vitesse de propagation à $6370 \mathrm{~m} / \mathrm{s}$. De plus le coefficient de qualité croit de27,1 à 31.1 pour une bande passante (f2-f1) à $-3 \mathrm{~dB}$ de 4,2 $\mathrm{MHz}$. Nous obtenons plusieurs plages de variation linéaire de la phase dans la bande passante de $128,45 \mathrm{MHz}$ à $132.65 \mathrm{MHz}$ (figure16). La phase est de $71,52^{\circ}$ à la fréquence de travaille sans effet de masse et elle décroit quand l'effet de masse augmente.

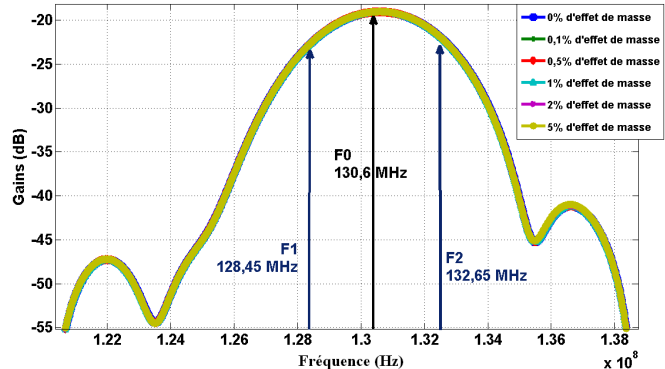

Figure 15. Gain sous effet de masse

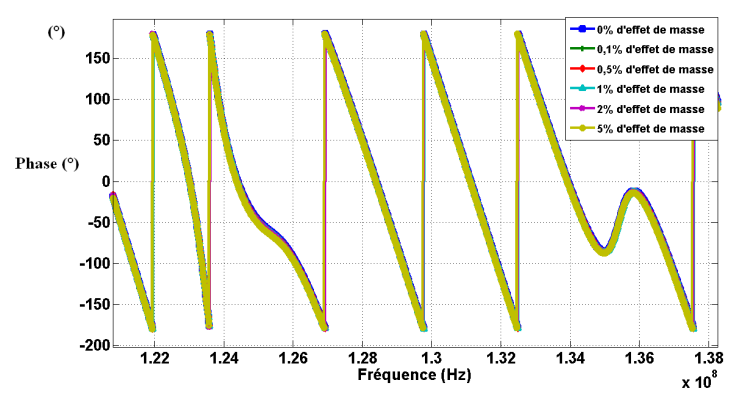

Figure 16. Phase sous effet de masse Grâce au zoom sur les courbes de phases autour de $0^{\circ}$, nous observons le décalage de la phase en fréquence (figure17).

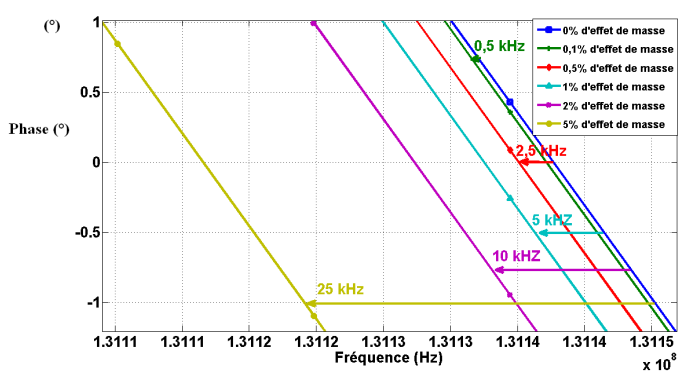

Figure 17. Variation de fréquence de la phase en fonction de l'effet de masse La variation en fréquence est de $0,5 \mathrm{kHz}, 2,5 \mathrm{kHz}, 5 \mathrm{kHz}, 10 \mathrm{KHz}, 25 \mathrm{KHz}$ pour un effet de masse de $0,1 \%, 0,5 \%, 1 \%, 2 \%$ et $5 \%$ respectivement. Cela montre que ce dispositif est plus précis et plus linéaire. 
L'étude de la sensibilité du dispositif (figure 18), montre que la variation de fréquence croit linéairement en fonction de la variation de la masse volumique de la couche sensible. Cela confirme une nouvelle fois que plus il y'a d'effet de masse, plus la concentration des particules à détecter est importante. Les études sur les dispositifs réels ont montré que la détection de $370 \mathrm{ppm}$ d'éthanol engendre une variation de fréquence de $500 \mathrm{~Hz}$ et cela correspond à un effet de masse de $0,18 \%$ [4]. Lorsqu' on fait le lien entre ces résultats et ceux de nos simulations, si la même concentration produit le même décalage fréquentiel, nous avons alors $0.1 \%$ d'effet de masse et la sensibilité serait de $0,74 \mathrm{ppm} / \mathrm{Hz}$, pour ce dispositif dans le cas d'une détection d'éthanol.

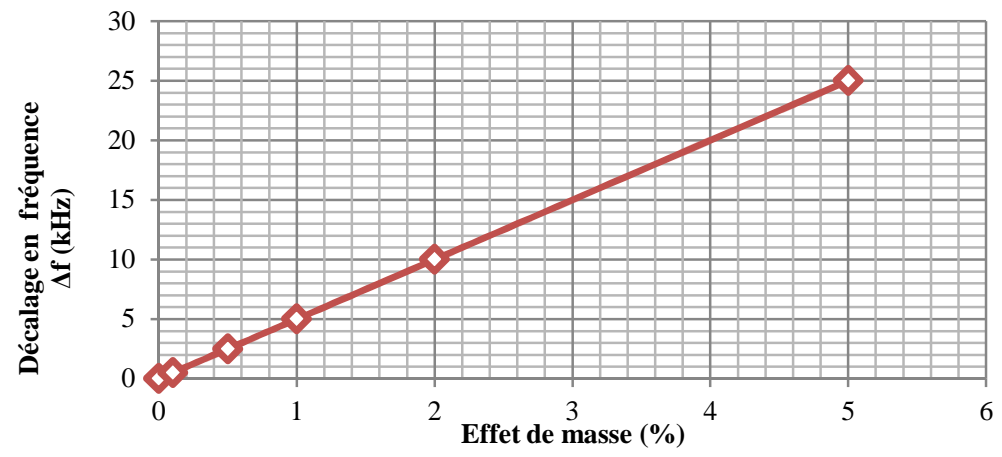

Figure 18. Variation de fréquence en fonction de l'effet de masse

\section{Conclusions et perspectives}

La modélisation et la simulation en 3D d'un capteur optimisé à ondes de Love, nous permet d'entrevoir et de confirmer l'influence du PDMS sur les dispositifs réels. Cette étude montre aussi l'importance des différents paramètres pour l'amélioration des capteurs par exemple le nombre de doigts sur chaque IDTs qui est très lié au coefficient de qualité des capteurs. Nous avons constaté que les structures simulées ont moins de pertes d'insertion $(21,7$ et $19 \mathrm{~dB})$ que les structures réelles (28 à $48 \mathrm{~dB}$ de pertes d'insertion). Nous obtenons une sensibilité de 5 $\mathrm{kHz}$ par pourcentage d'effet de masse avec le modèle intégrant le PDMS. Nous mettons en perspective l'exploration de nouvelles structures avec des nouveaux matériaux et/ou des géométries différentes, puis plus complexes.

\section{Références}

[1] D. Rebiere, « Microsystèmes à ondes acoustiques de surface dédiés à la détection (bio) chimique: Exemples d'applications», http://extranet.innovalisaquitaine.org/RICTIC2007/Rebiere.pdf

[2] C. Zimmermann, "Conception, réalisation et étude de microcapteurs à ondes de Love pour applications en milieu gazeux. Cas de la propagation de composés organophosphorés », Thèse de doctorat $\mathrm{n}^{\circ}$ 2471, Université Bordeaux I, (2002).

[3] O Tamarin, R Djoumer, H Hallil, D Rebière, C Dejous « Modélisation par Eléments Finis d'une Ligne à Retard à Ondes de Love » Actes CFM 2013.

[4] Tortissier, G.; Blanc, L.; Tetelin, A.; Zimmermann, C.; Lachaud, J.-L.; Boissière, C.; Sanchez, C.; Dejous, C.; Rebière, D. « Mesoporous Coated Films on Love Wave Acoustic Devices for Gas Detection », Sensor Letters, Volume 7,pp. 984-988(5) 
[5] David Rabus, «Résonateur à ondes élastiques de volume à modes harmoniques élevés (HBARs) pour mesures gravimétriques : Application à la détection de gaz », (2013)

[6] Vincent Raimbault, «Étude et développement d'un système microfluidique à ondes de Love dédié à la caractérisation de fluides complexes », (2008)

[7] Hakim Tarbague, «Étude et mise au point d'une plateforme de biodétection de microorganismes couplant immunocapteur à ondes de Love et dispositifs PDMS microfluiques», (2011)

[8] http://www.mit.edu/ 6.777/matprops/pdms.htm

\section{Biographie des auteurs}

Hamida HALLIL est Enseignante Chercheuse à l'université de Bordeaux depuis 2011. Elle assure des enseignements d'électronique analogique et numérique, microsystèmes et automatismes. Ses activités de recherche sont basées au sein du laboratoire IMS (de l'intégration du matériau au système). Sa thématique de recherche est principalement axée sur l'étude et le développement de plates formes de détection innovantes pour des applications environnementales et médicales.

Prince BAHOUMINA est doctorant en électronique en première année à l'université de Bordeaux en partenariat avec le laboratoire IMS de Bordeaux. Il travaille sur les capteurs de gaz flexible ultra-sensibles à transduction électromagnétique avec des matériaux carbonés.

Ollivier TAMARIN est enseignant chercheur à l'Université des Antilles et de la Guyane. Il est directeur de l'IUT de de Kourou. Il assure des enseignements d'électronique analogique et numérique au département GEII. Sa thématique de recherche est axée sur les capteurs à ondes acoustiques pour des applications environnementales et médicales.

Corinne DEJOUS est Professeur des Universités à Bordeaux-INP / ENSEIRB-MATMECA depuis 2009.

Elle est directrice de la filière Systèmes Electroniques Embarqués en alternance. Ses activités de recherche sont basées au sein du laboratoire IMS et Sa thématique de recherche est principalement axée sur l'étude et le développement de plates formes de détection innovantes pour des applications environnementales et médicales.

Dominique REBIERE est Professeur des Universités à l'université de Bordeaux. Il est Délégué régional à la recherche et à la technologie (DRRT) pour la région Aquitaine depuis 2013. Ses activités de recherche sont basées au sein du laboratoire IMS et Sa thématique de recherche est principalement axée sur l'étude et le développement de plates formes de détection innovantes pour des applications environnementales et médicales. 